ANNALES

POLONICI MATHEMATICI

IX (1960)

\title{
Propriétés d'une classe de fonctions holomorphes aux fonctions limites discontinues
}

\author{
par W. Pogonzelski (Warszawa)
}

1. Introduction. Soit dans le plan de la variable complexe un domaine $S^{+}$limité par les courbes fermées $L_{0}, L_{1}, \ldots, L_{n}(n \geqslant 0)$. On admet que les courbes $L_{1}, \ldots, L_{n}$ limitent les domaines bornés disjoints $S_{1}^{-}, \ldots, S_{n}^{-}$ et que la courbe $L_{0}$ renferme toutes les courbes $L_{1}, \ldots, L_{n}$. Nous désignerons par $S_{0}^{-}$le domaine non borné, situé à l'extérieur de la courbe $L_{0}$, par $L$ et $S^{-}$les sommes suivantes d'ensembles de pointis:

$$
L=L_{0}+L_{1}+\ldots+L_{n}, \quad S^{-}=S_{0}^{-}+S_{1}^{-}+\ldots+S_{n}^{-} .
$$

Remarquons que la ligne $L_{0}$ peut ne pas exister; dans ce cas le domaine $S^{+}$s'étendra à l'infini. Nous admettons que les lignes $L_{0}, \ldots, L_{n}$ ont des tangentes continues en tout point, sauf sur une suite finie de points $c_{1}, c_{2}, \ldots, c_{p}$, situés sur une au moins des lignes $L_{0}, \ldots, L_{n}$, qui sont des points anguleux ou de rebroussement de ces courbes. On admet ensuite que le sens positif des lignes $L_{1}, \ldots, L_{n}$ coïncide avec le sens négatif de rotation dans le plan de la variable complexe et que le sens positif de la ligne $L_{0}$ cö̈ncide avec le sens positif dans ce plan.

Conformément à la définition que nous avons donnée dans les travaux [1] nous appellerons classe

$$
\mathfrak{S}_{\alpha}^{h}(0 \leqslant \alpha<1,0<h<1, \alpha+h<1)
$$

l'ensemble de toutes les fonctions complexes $\varphi(t)$, définies sur $L$, qui sont continues en tout point $t$ différent des points de discontinuité $c_{1}, \ldots, c_{p}$ et vérifient les inégalités

$$
\begin{gathered}
|\varphi(t)|<\frac{\text { const }}{\prod_{\nu=1}^{p}\left|t-c_{\nu}\right|^{\alpha}}, \\
\left|\varphi(t)-\varphi\left(t_{1}\right)\right|<\frac{\text { const }\left|t-t_{1}\right|^{h}}{\left[\left|t-c_{\nu}\right|\left|t_{1}-c_{\nu_{1}}\right|\right]^{a+h}},
\end{gathered}
$$


où $t$ et $t_{1}$ désignent deux points arbitraires d'une même ligne $L_{j}$, situés à l'intérieur d'un arc $\widehat{c_{\nu} c_{\nu_{1}}}$ sur cette courbe, ne contenant pas d'autre point de discontinuité et tels que $t_{1} \in t c_{\nu_{1}}$.

Dans le cas où une courbe $L_{j}$ ne contient qu'un seul point de discontinuité $c_{v}$, on admet que le dénominateur de la seconde des inégalités (1) ne contient qu'un seul facteur $\left|t-c_{\nu}\right|$ tel que $\left|t-c_{\nu}\right| \leqslant\left|t_{1}-c_{\nu}\right|$ et, en outre, que la longueur de l'are $\bar{t}_{1}$, qui ne contient pas le point $c_{y}$, est moindre qu'un nombre positif inférieur à la longueur totale de la courbe $L_{j}$. Dans le cas où une courbe $L_{j}$ ne contient aucun point de discontinuité, le dénominateur dans la seconde des inégalités (1) ne contient pas les facteurs $\left|t-c_{\nu}\right|$ et $\left|t_{1}-c_{\nu_{1}}\right|$.

Les fonctions discontinues de classe $\mathfrak{S}_{\alpha}^{h}$ ont les propriétés suivantes, qui jouent un rôle important dans la théorie des problèmes aux limites discontinues de la théorie des fonctions analytiques.

Propriété 1. Si la fonction $\varphi(t)$ est de classe $\mathfrak{S}_{\alpha}^{h}$ relativement aux points de discontinuité $c_{1}, c_{2}, \ldots, c_{p}$ sur les courbes $L$, la fonction $\psi(t)$, définie par l'intégrale singulière de Cauchy

$$
\psi(t)=\int_{\bar{L}} \frac{\varphi(\tau) d \tau}{\tau-t}
$$

en tout point $t \neq c_{y}$ des courbes $L$, est de classe $\mathfrak{G}_{\varrho}^{h}$ oì $\varrho=\alpha$ si $\alpha>0$ et $\varrho$ est un nombre positif arbitrairement petit si $\alpha=0$.

ProprIÉté 2. Si la fonction $\varphi(t)$ est de classe $\mathfrak{S}_{\alpha}^{h}$ sur $L$, la fonction

$$
\Phi(z)=\int_{L} \frac{\varphi(\tau) d \tau}{\tau-z}
$$

holomorphe séparément à l'intérieur des domaines $S^{+}, S_{0}^{-}, S_{1}^{-}, \ldots, S_{n}^{-}$, vérifie l'inégalité à faible singularité

$$
|\Phi(z)|<\frac{\text { const }}{\left|z-c_{\nu}\right|^{e}}
$$

(@ ayant la valeur fixée précédemment) au voisinage de tout point de discontinuité $c_{p}$ et ses fonctions limites

$$
\Phi^{ \pm}(t)=\lim _{z \rightarrow t} \Phi(z),
$$

relatives aux domaines $S^{+}$et $S^{-}$, appartiennent à la classe $\mathfrak{S}_{0}^{h}$ sur les courbes $L$.

Nous avons donné les démonstrations de ces propriétés dans les travaux [1].
DÉfinition 1. Nous appelons classe $\mathfrak{S}_{a}^{h}\left(c_{k_{1}}, \ldots, c_{k_{q}}\right)(\alpha>0)$ l'ensemble de toutes les fonctions $\varphi(t)$ de classe $\mathfrak{S}_{\alpha}^{h}$ qui sont bornées, ou presque bornées, dans les voisinages des points de discontinuité arbitrairement distingués $c_{k_{1}}, \ldots, c_{k_{q}}(q \leqslant p)$, e'est-à-dire vérifiant des inégalités de la forme

$$
|\varphi(t)|<\frac{\text { const }}{\prod_{\nu=1}^{p}\left|t-c_{\nu}\right|^{e_{\nu}}}, \quad\left|\varphi(t)-\varphi\left(t_{1}\right)\right|<\frac{\text { const }\left|t-t_{1}\right|^{h}}{\left|t-c_{\nu}\right|^{e_{\nu}+h}\left|t_{1}-c_{\nu_{1}}\right|^{e_{\nu_{1}}+h}}
$$

où $\varrho_{v}=0$ ou est un nombre positif arbitrairement petit, si $v=k_{1}, \ldots, k_{q}$ et $\varrho_{v}=\alpha$, pour les autres points de discontinuité $e_{v}$.

La définition 1 est un peu généralisée par rapport à celle donnée dans notre travail [1]. Cette généralisation permet de conférer une plus grande symétrie aux propriétés et aux problèmes considérés ultérieurement. Signalons que l'ensemble $c_{k_{1}}, \ldots, c_{k_{q}}$ peut être vide.

PROPRIÉtÉ 3. Si la fonction $\phi(t)$ appartient à la classe $\mathfrak{S}_{a}^{h}\left(c_{k_{1}}, \ldots, c_{k_{q}}\right)$, la fonction $\psi(t)$, définie par l'intégrale singulière de Cauchy (2), appartient aussi à la même classe.

Cette propriété résulte de la propriété 1.

Remarque. Nous appelons classe $\mathfrak{S}_{\alpha}\left(c_{k_{1}}, \ldots, c_{k_{q}}\right)$ l'ensemble de toutes les fonctions de classe $\mathfrak{S}_{a}^{h}\left(c_{k_{1}}, \ldots, c_{k_{q}}\right)$ qu'on obtient en admettant pour paramètre $h$ toutes les valeurs telles que $0<\alpha+h<1$. On définit d'une façon analogue la classe $\mathfrak{S}\left(c_{k_{1}}, \ldots, c_{k_{q}}\right)$.

PROPRIÉté 4. Si la fonction donnée $\psi(t)$ appartient à la classe $\mathfrak{S}_{a}^{h}\left(c_{k_{1}}, \ldots, c_{k_{q}}\right)$, la fonction $\varphi(\tau)$, vérifiant l'équation $(2)$ en tout point $t \neq c_{\nu}$ des lignes $L$, existe et elle est donnée par la formule

$$
\varphi(t)=-\frac{1}{\pi^{2}} \int_{L} \frac{\psi(\tau) d \tau}{\tau-t}
$$

elle est donc aussi de classe $\mathfrak{S}_{a}^{h}\left(c_{k_{1}}, \ldots, c_{k_{q}}\right)$ et unique dans la classe $\mathfrak{S}\left(c_{k_{1}}, \ldots, c_{k_{q}}\right)$.

On démontre cette propriété par un raisonnement analogue à celui qu'on applique aux fonctions vérifiant une simple condition de Hölder.

2. Fonctions holomorphes de classe $H_{a}^{h}$ et leurs propriétés. DÉfinition 2. Nous appelons classe $H_{\alpha}^{h}$ l'ensemble de toutes les fonctions $\Phi(z)$, holomorphes ou séparément holomorphes dans les domaines $S^{+}$, $S_{0}^{-}, S_{1}^{-}, \ldots, S_{n}^{-}$, dont les fonctions limites

$$
\Phi^{ \pm}(t)=\lim _{z \rightarrow t} \Phi(z) \quad\left(t \neq c_{\nu}\right)
$$


sont de classe $\mathfrak{S}_{a}^{h}$ sur l'ensemble $L$, et qui vérifient l'inégalité à singularité faible

$$
|\Phi(z)|<\frac{\text { const }}{\left|z-c_{\nu}\right|^{\theta}}
$$

dans les voisinages de tous les points de discontinuité $c_{p} ; \theta$ est un nombre positif inférieur à l'unité, non fixé.

Observons que la classe $H_{a}^{h}$ est un sous-ensemble de la classe $H_{a_{1}}^{h_{1}}$ $\left(\alpha_{1}+h_{1}<1\right)$ si $\alpha \leqslant \alpha_{1}, h_{1} \leqslant h$. Cette propriété résulte immédiatement de la propriété analogue de la classe $\mathfrak{S}_{\alpha}^{h}$ que nous avons démontré dans le travail [1].

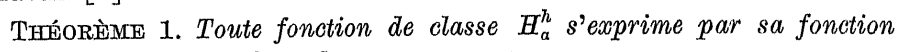
limite au moyen de la formule

$$
\Phi(z)=\frac{1}{2 \pi i} \int_{\dot{L}} \frac{\Phi^{+}(\tau) d \tau}{\tau-z}
$$

si $z \in S^{+}$et par la formule

$$
\Phi(z)=-\frac{1}{2 \pi i} \int_{L} \frac{\Phi^{-}(\tau) d \tau}{\tau-z}+\Phi(\infty) \varepsilon_{j}
$$

si $z \epsilon S_{j}^{-}\left(\varepsilon_{0}=1, \varepsilon_{1}=\varepsilon_{2}=\ldots=\varepsilon_{n}=0\right)$, si l'on suppose l'existence de la courbe $L_{0}$ et la régularité de la fonction $\Phi(z)$ à l'infini. Dans le cas où il n'y a pas de contour $L_{0}$, on a les formules

$$
\begin{gathered}
\Phi(z)=\frac{1}{2 \pi i} \int_{L} \frac{\Phi^{+}(\tau) d \tau}{\tau-z}+\Phi(\infty), \quad \text { si } \quad z \in S^{+}, \\
\Phi(z)=\frac{-1}{2 \pi i} \int_{L} \frac{\Phi^{-}(\tau) d \tau}{\tau-z}, \quad \text { si } \quad z \in S^{-}
\end{gathered}
$$

Démonstration. On prouve facilement le théorème en entourant tout point de discontinuité $c_{v}$ d'une circonférence $C_{v}$ de rayon $\varepsilon$ suffisamment petit pour qu'elle ne contienne pas le point $z$ du domaine $S^{\text {t }}$ ou $S^{-}$et qu'elle ne coupe la ligne $L$ qu'en deux points.

D'après le théorème classique de Cauchy, nous aurons alors (en supposant, pour fixer les idées, que $z \in S^{+}$)

$$
\Phi(z)=\frac{1}{2 \pi i} \int_{L-\Sigma \widehat{a_{v} b_{v}}} \frac{\Phi^{+}(\tau) d \tau}{\tau-z}+\frac{1}{2 \pi i} \sum_{p=1}^{p} \int_{\gamma_{\nu}} \frac{\Phi(\tau) d \tau}{\tau-z}
$$

où $\widehat{a_{y} b_{v}}$ sont les parties des courbes $L$ situées à l'intérieur des cercles $C_{v}$ et $\gamma_{v}$ - les ares des cercles qui joignent les points $a_{v}, b_{v}$ et sont contenus dans $S^{+}$. En vertu des inégalités admises (6) et (1), les intégrales étendues aux arcs $\gamma_{\nu}$ tendent vers zéro, si le rayon $\varepsilon \rightarrow 0$, et la première des intégrales (9) tend vers une limite déterminée, si $\varepsilon \rightarrow 0$; nous aurons donc la conclusion (7). Les autres cas se traitent d'une façon analogue.

DÉfinition 3. Nous appelons classe $H_{\alpha}^{h}\left(c_{k_{1}}, \ldots, c_{k_{u}}\right)$ l'ensemble de toutes les fonctions $\Phi(z)$ de classe $H_{a}^{h}$ dont les fonctions limites $\Phi^{ \pm}(t)$ appartiennent à la classe $\mathfrak{S}_{a}^{h}\left(c_{k_{1}}, \ldots, c_{k_{q}}\right)$. Observons que l'ensemble des points distingués $c_{k_{1}}, \ldots, c_{k_{2}}$ peut être vide. Dans ce cas la nouvelle classe est identique à la classe $H_{\alpha}^{h}$. Nous appelons classe $H_{a}\left(c_{k_{1}}, \ldots, c_{k_{q}}\right)$ l'ensemble de toutes les fonctions de classe $H_{a}^{h}\left(c_{k_{1}}, \ldots, c_{k_{q}}\right)$ qu'on obtient en admettant pour paramètre $h$ toutes les valeurs positives telles que $0<a+h<1$. Par analogie on définit la classe $H\left(c_{k_{1}}, \ldots, c_{k_{q}}\right)$.

THÉORÈme 2. Toute fonction $\Phi(z)$ de classe $H_{\alpha}^{h}\left(c_{k_{1}}, \ldots, c_{k_{q}}\right)(\alpha>0)$ vérifie dans les voisinages des points de discontinuité $c_{p}$ une inégalité

$$
|\Phi(z)|<\frac{\text { const }}{\left|z-c_{y}\right|^{p_{y}}}
$$

où $\varrho_{\nu}=\alpha$, si $v \neq k_{1}, \ldots, k_{q}$, et $\varrho_{\nu}$ est un nombre positif arbitrairement petit, si $v=k_{1}, \ldots, k_{q}$. Cette propriété résulte immédiatement de la définition de la classe $H_{a}^{h}\left(c_{k_{1}}, \ldots, c_{k_{q}}\right)$, du théorème 1 et de la propriété (4) des fonctions de classe $\mathfrak{S}_{\alpha}^{h}$.

THÉORÈME 3. Si la fonction $\varphi(t)$, définie sur $L$, est de classe $\mathfrak{S}_{a}^{h}\left(c_{k_{1}}, \ldots, c_{k_{q}}\right)(\alpha>0)$, la fonction définie par l'intégrale

$$
\Phi(z)=\frac{1}{2 \pi i} \int_{L} \frac{\varphi(\tau) d \tau}{\tau-z},
$$

pour $z \in S^{+}+S^{-}$, appartient à la classe $H_{\alpha}^{h}\left(c_{k_{1}}, \ldots, c_{k_{q}}\right)$.

Démonstration. La fonction $\varphi(\tau)$ vérifie une simple condition de Hölder dans toute partie fermée des ares $L$ ne eontenant pas le points de discontinuité $c_{\nu}$.

Donc, d'après les formules de Plemelj, la fonction (11) admet des valeurs limites des deux côtés des lignes $L$

$$
\lim _{z \rightarrow t} \Phi(z)=\Phi^{ \pm}(t)= \pm \frac{1}{2} \varphi(t)+\frac{1}{2 \pi i} \int_{L} \frac{\varphi(\tau) d \tau}{\tau-t}
$$

en tout point $t \neq c_{\nu}$ sur $L$. Le signe + se rapporte au domaine $S^{+}$et le signe - au domaine $S^{-}$. II en résulte, en vertu de la propriété 3 , que les fonctions limites (12) appartiennent à la classe $\mathfrak{S}_{\alpha}^{h}\left(c_{k_{1}}, \ldots, c_{k_{q}}\right)$. D'autre Annales Polonici Mathematici IX. 
part, en vertu de l'inégalité ( $\left.1^{\prime}\right)$ et de la propriété 2 , la fonction (11) vérifie une inégalité de la forme

$$
|\Phi(z)|<\frac{\text { const }}{\prod_{\nu=1}^{p}\left|\dot{z}-c_{\nu}\right|^{e_{\nu}}},
$$

donc elle appartient à la classe $H_{a}^{h}\left(c_{k_{1}}, \ldots, c_{k_{q}}\right)$ c. q. f. d.

THÉoRìme 4. A. Pour que la fonction $\varphi(t)$ de classe $\mathfrak{S}_{\alpha}^{h}\left(c_{k_{1}}, \ldots, c_{k_{\alpha}}\right)$ $(\alpha>0)$, donnée en tout point $t \neq c_{v}$ sur $L$, soit fonction limite d'une fonction holomorphe dans $S^{+}$de classe $\mathfrak{H}_{a}^{h}\left(c_{k_{1}}, \ldots, c_{k_{q}}\right.$ ) (nulle à l'infini dans le cas où il n'y a pas de contour $\left.L_{0}\right)$, il faut et il suffit que la fonction donnée $\varphi(t)$ vérifie une équation intégrale

$$
-\varphi(t)+\frac{1}{\pi i} \int_{L} \frac{\varphi(\tau) d \tau}{\tau-t}=0
$$

en tout point $t$ sur $L$ different des points $c_{1}, c_{2}, \ldots, c_{p}$.

B. Pour que la fonction $\varphi(t)$ de classe $\mathfrak{S}_{a}^{h}\left(c_{k_{1}}, \ldots, c_{k_{q}}\right)(\alpha>0)$, donnée en tout point $t \neq c_{v}$ sur $L$, soit fonction limite d'une fonction séparément holomorphe dans $S^{-}$de classe $H_{a}^{h}\left(c_{k_{1}}, \ldots, c_{k_{q}}\right)$ (nulle à l'infini dans le cas où le contour $L_{0}$ existe,) il faut et il suffit que la fonction donnée $p(t)$ vérifie une équation intégrale

$$
+\varphi(t)+\frac{1}{\pi i} \int_{L} \frac{\varphi(\tau) d \tau}{\tau-t}=0
$$

en tout point $t$ sur $L$ différent des points $c_{\nu}$.

Démonstration. Pour démontrer la partie $\mathrm{A} d u$ théorème, remarquons que, s'il existe une fonction holomorphe $\Phi(z) \epsilon H_{\alpha}^{h}$ dans $S^{+}$ (nulle à l'infini) telle que

$$
\lim _{z \rightarrow t} \Phi(z)=\Phi^{+}(t)=\varphi(t) \quad\left(t \neq c_{v}\right)
$$

celle-ci s'exprime, d'après le théorème 1, par la formule

$$
\Phi(z)=\frac{1}{2 \pi i} \int_{L} \frac{\varphi(\tau) d \tau}{\tau-z}
$$

en tout point $z \epsilon S^{+}$. Il en résulte, d'après les formules de Plemelj (12), la relation (13).

Inversoment, si la relation (13) est satisfaite en tout point $t \neq c_{\nu}$ de $L$, la fonction (13") admet, en vertu des formules (12), les valours limites

$$
\lim _{z \rightarrow t} \Phi(z)=\Phi^{+}(t)=\varphi(t)
$$

pour $t \neq c_{y}$ et appartient à la classe $H_{a}^{h}\left(c_{k_{1}}, \ldots, c_{k_{q}}\right)$, d'après le théorème 3 . Cette fonction est unique dans la classe $H_{a}^{h}$. La seconde partie B du théorème se démontre d'une façon analogue.

THÉóRÈme 5. Si la dérivée d'une fonction $\Phi(z)$, holomorphe séparément dans les domaines $S^{+}, S_{0}^{-}, S_{1}^{-}, \ldots, S_{n}^{-}$, appartient $\grave{a}$ la classe $H_{\alpha}^{h}$ :

$$
\Phi^{\prime}(z) \in H_{\alpha}^{h}
$$

la fonction $\Phi(z)$ elle-même admet les valeurs limites $\Phi^{ \pm}(t)=\lim _{z \rightarrow t} \Phi(z)$ en tout point $t$ des courbes $L$ des deux côtés de celles-ci, même aux points de discontinuité $c_{1}, c_{2}, \ldots, c_{p}$, et les fonctions limites vérifient une condition de Hölder

$$
\left|\Phi^{ \pm}\left(t_{1}\right)-\Phi^{ \pm}\left(t_{2}\right)\right|<\mathrm{const}\left|t_{1}-t_{2}\right|^{\theta}
$$

pour tous les points des lignes $L$, où $\theta=1-\alpha$, si $\alpha>0$, et $\theta$ est un nombre positif arbitraire inférieur à l'unité, si $\alpha=0$.

Démonstration. Pour prouver l'existence d'une fonction limite, par exemple $\Phi^{+}(t)$, il suffit de démontrer l'existence d'une limite de la fonction $\Phi(z)$, si le point $z \in S^{+}$tend d'une façon arbitraire vers un point de discontinuité $c_{v} \in L$. Soit done un arc arbitraire $\widehat{z}_{0} c_{\nu}$, à tangentes continues, joignant un point $z_{0} \in S^{+}$à un point de discontinuité $c_{\nu}$ et contenu à l'intérieur du domaine $S^{+}$. Nous avons alors

$$
\Phi(z)=\Phi\left(z_{0}\right)+\int_{z_{0}}^{z} \Phi^{\prime}(\zeta) d \zeta,
$$

$z$ étant un point arbitraire à l'intérieur de l'are $\widetilde{z_{0} c_{\nu}}$. Il en résulte, en vertu de la propriété (6) des fonctions de classe $H_{a}^{h}$, que la fonction $\Phi(z)$ tend vers une limite déterminée

$$
\lim _{z \rightarrow c_{v}} \Phi(z)=\Phi^{+}\left(c_{v}\right)=\Phi\left(z_{0}\right)+\int_{z_{0}}^{c_{v}} \Phi^{\prime}(\zeta) d \zeta
$$

si le point $z$ tend vers un point de discontinuité $c_{\nu}$. L'intégrale absolument convergente dans la formule (14) ne dépend pas de la forme de l'arc $\widetilde{z_{0} c_{v}}$ et la limite (14) ne dépend pas du choix du point $z_{0}$. Pour étudier la continuité de la fonction limite $\Phi^{+}(t)$, remarquons que

$$
\Phi^{+}(t)-\Phi^{+}\left(c_{\nu}\right)=\int_{c_{\nu}}^{t} \Phi^{\prime}(\zeta) d \zeta
$$

l'intégrale étant prise le long d'un are $\widehat{e_{\nu} t}$ sur $L . \Pi$ en résulte, d'après le théorème (2),

$$
\left|\Phi^{+}(t)-\Phi^{+}\left(c_{\nu}\right)\right|<\mathrm{const} \int_{c_{\nu}}^{t} \frac{d l_{\zeta}}{\left|\zeta-c_{\nu}\right|^{e_{\nu}}}<\mathrm{const}\left|t-c_{\nu}\right|^{1-e_{\nu}}
$$


d'où l'on déduit la conclusion du théorème 5. Celle-ci admet une exception dans le cas où les points $t_{1}$ et $t_{2}$ sont situés des deux côtés d'un point de rebroussement $c_{v}$; au lieu de la différence $t_{1}-t_{2}$ il faut alors substituer la différence $s_{1}-s_{2}$ des coordonnées curvilignes des points $t_{1}$ et $t_{2}$.

THÉorł̀me 6. Si la fonction $\varphi(t)$, définie sur $L$, est de classe $\mathfrak{S}_{\alpha}^{h}\left(c_{k_{1}}, \ldots, c_{k_{q}}\right)$, toutes les fonctions $\Phi(z)$ de classe $H_{\alpha}^{h}\left(c_{k_{1}}, \ldots, c_{k_{q}}\right)$ pour lesquelles la différence des valeurs limites a une valeur donnée

$$
\Phi^{+}(t)-\Phi^{-}(t)=\varphi(t)
$$

en tout point $t \neq c_{y}$ sur $L$, sont exprimées par la formule

$$
\Phi(z)=\frac{1}{2 \pi i} \int_{\dot{L}} \frac{\varphi(\tau) d \tau}{\tau-z}+P(z),
$$

où $P(z)$ est une fonction entière arbitraire.

Démonstration. D'après les propriétés citées 1 et 2 , toute fonction (15) est de classe $H_{a}^{h}\left(c_{k_{1}}, \ldots, c_{k_{q}}\right)$; en outre, en vertu de formule de Plemelj, elle admet des valeurs limites vérifiant la relation (14') en tout point $t \neq c_{y}$ de $L$. Pour démontrer que la formule (15) comprend l'ensemble de toutes les fonctions de classe $H_{\alpha}^{h}\left(c_{k_{1}}, \ldots, c_{k_{q}}\right)$ vérifiant la condition (14'), supposons que $\Psi(z)$ soit une fonction quelconque de classe $H_{\alpha}^{h}\left(c_{k_{1}}, \ldots, c_{k_{q}}\right)$ vérifiant la relation

$$
\Psi^{+}(t)-\Psi^{-}(t)=\varphi(t) .
$$

Il en résulte que la différence $\Omega(z)=\Phi(z)-\Psi(z)$ est une fonction de classe $H_{\alpha}^{h}\left(c_{k_{1}}, \ldots, c_{k_{q}}\right)$, dont les valeurs limites des deux côtés de toute ligne sont égales: $\Omega^{+}(t)=\Omega^{-}(t)$ en tout point $t$ de $L$ différent des points de discontinuité $c_{\nu}$. La fonction $\Omega(z)$ est donc holomorphe dans tout le plan, excepté au plus aux points isolés $c_{v}$. Mais, d'après l'hypothèse, cette fonction vérifie dans les voisinages de ces points l'inégalité

$$
|\Omega(z)|<\frac{\text { const }}{\left|z-c_{y}\right|^{\theta}} \quad(0 \leqslant \theta<1)
$$

à faible singularité, donc ces points sont réguliers et la fonction $\Omega(z)$ est entière c. q. f. d.

THÉORÈME 7. Si la fonction $\varphi(\tau)$ appartient d̀ la classe $\mathfrak{S}_{\alpha}^{h}\left(c_{k_{1}}, \ldots, c_{k_{q}}\right)$, la fonction $\Psi(t)$, donnée par l'intégrale singulière au sens de Cauchy

$$
\Psi(t)=\prod_{j=1}^{p}\left(t-c_{j}\right)^{\gamma_{j}} \int_{i} \frac{\varphi(\tau) d \tau}{\prod_{j=1}^{p}\left(\tau-c_{j}\right)^{\gamma_{j}}(\tau-t)}
$$

en tout point $t \neq c_{v}$ sur $L$, est de classe $\mathfrak{H}_{\sigma}^{h}\left(c_{k_{1}}, \ldots, c_{k_{q}}\right)$; on admet que les constantes $\gamma_{j}=\gamma_{j}^{\prime}+i \gamma_{j}^{\prime \prime}$ vérifient les inégalités

$$
\begin{array}{ccl}
0 \leqslant \gamma_{j}^{\prime}<1, & \text { si } & j=k_{1}, \ldots, k_{q}, \\
-1<\gamma_{j}^{\prime} \leqslant 0, & \text { si } & j \neq k_{1}, \ldots, k_{q}, \\
h+\max \left|\gamma_{j}^{\prime}\right|<1, & \text { si } & j=1,2, \ldots, p,
\end{array}
$$

la constante $\sigma$ a les valeurs suivantes:

$$
\sigma=\left\{\begin{array}{lll}
\alpha, & \text { si } & \alpha>\max \left|\gamma_{j}^{\prime}\right| \\
\max \left|\gamma_{j}^{\prime}\right|, & \text { si } & \alpha<\max \left|\gamma_{j}^{\prime}\right| ;
\end{array} \quad\left(j \neq k_{1}, \ldots, k_{q}\right),\right.
$$

en outre on a fixé les branches continues des fonctions $\left(t-c_{j}\right)^{\gamma_{j}}$ sur tout arc composant de $L$.

Le théorème est une conséquence de la propriété 3.

3. Théorèmes sur certaines représentations intégrales des fonctions holomorphes $H_{\alpha}^{h}$. Nous établirons maintenant deux théorèmes sur les représentations intégrales des fonctions de classe $H_{a}^{h}$, qui sont une extension des théorèmes démontrés par une analyse savante par Vécoua [2] (voir aussi la monographie de Mouskhelichvili [3], pages 200-209). Soit dans le plan de la variable complexe une seule courbe fermée $L$ limitant le domaine intériour $S^{+}$. Soit encore une suite finie de points $c_{1}, c_{2}, \ldots, c_{p}$ sur cette courbe $L$ rangés dans un ordre qui correspond au sens positif de cette courbe, coïncidant avec le sens positif de rotation dans le plan de la variable complexe. On admet que la courbe $L$ a en tout point, même aux points $c_{1}, c_{2}, \ldots, c_{p}$, des tangentes continues qui font avec une direction fixée un angle vérifiant une condition de Hölder.

On admet notamment que l'angle $\Delta\left(t, t_{1}\right)$ entre les tangentes en deux points arbitraires $t$ et $t_{1}$ de la courbe $L$ vérifie l'inégalité

$$
\left|\Delta\left(t, t_{1}\right)\right|<\text { const }\left|t-t_{1}\right|^{h_{L}}
$$

$h_{L}$ étant une constante positive, non supérieure à l'unité.

THÉORÈME 8. Toute fonction $\Phi(z)$ holomorphe dans le domaine $\mathrm{S}^{+}$, appartenant à la classe $B_{a}^{h}\left(c_{k_{1}}, \ldots, c_{k_{q}}\right)$ relativement aux points de discontinuité $c_{1}, \ldots, c_{p}$ sur $L$, peut être exprimée d'une façon unique par la formule intégrale

$$
\Phi(z)=\int_{L} \frac{\mu(\tau) d \sigma}{1-z / \tau}+i C
$$

où $\mu(\tau) \epsilon \mathfrak{S}_{a}^{h^{\prime \prime}}\left(c_{k_{1}}, \ldots, c_{k_{q}}\right)$ est une fonction réelle définie sur $L, O$ est une constante réelle, $\sigma$ désigne l'abscisse curviligne du point d'intégration $\tau$, 
l'origine $z=0$ est contenue à l'intérieur du domaine $S^{+}$, enfin la fonction $\mu(\tau)$ et la constante $C$ sont définies d'une façon unique pour la fonction donnée $\Phi(z)$ (on a désigné $h^{\prime}=\min \left(h, h_{L}\right)$ ).

Démonstration. On obtient la preuve du théorème 8 en s'appuyant sur les propriétés des fonctions des classes $\mathfrak{S}_{a}^{h}$ et $H_{\alpha}^{h}$ et en suivant d'une façon abrégée un raisonnement analogue à celui qui à été donné dans la monographie [3]. En posant

$$
\tau^{\prime}=\frac{d \tau}{d \sigma}=\frac{d x}{d \sigma}+i \frac{d y}{d \sigma}, \quad \bar{\tau}^{\prime}=\frac{d x}{d \sigma}-i \frac{d y}{d \sigma}=\tau^{\prime-1},
$$

nous pouvons écrire la formule demandée (17) sous la forme

$$
\Phi(z)=\frac{1}{2 \pi i} \int_{\bar{L}} \frac{2 \pi i \mu(\tau) \tau \bar{\tau}^{\prime}+i C}{\tau-z} d \tau .
$$

En tenant maintenant compte de la représentation (7) d'une fonction donnée $\Phi(z)$ de classe $H_{a}^{h}\left(c_{k_{1}}, \ldots, c_{k_{\alpha}}\right)$ et du théorème 3 , nous pouvons affirmer que si la représentation (17) est vraie, il doit exister une fonction $\Omega(z)$ de classe $H_{a}^{h^{\prime}}\left(c_{k_{1}}, \ldots, c_{k_{q}}\right)$, holomorphe dans le domaine extérieur $S^{-}$et nulle à l'infini, telle qu'on ait

$$
2 \pi i \mu(\tau) \tau \bar{\tau}^{\prime}+i C=\Phi^{+}(\tau)-\Omega^{-}(\tau) .
$$

En introduisant la nouvelle fonction inconnue

$$
\Omega_{0}(z)=\Omega(z)+i C,
$$

nous voyons que cette fonction doit vérifier la condition limite

$$
\mathrm{re}\left[\frac{1}{\tau \bar{\tau}^{\prime}} \Omega_{0}^{-}(\tau)\right]=\operatorname{re}\left[\frac{1}{\tau \bar{\tau}^{\prime}} \Phi^{+}(\tau)\right]=c(\tau)
$$

et admettre une valeur purement imaginaire à l'infini. La fonction $\Omega_{0}(z)$ de classe $H_{a}^{h \prime}\left(c_{k_{1}}, \ldots, c_{k_{q}}\right)$ est done une solution du problème aux limites discontinu de Riemann dans le domaine $S^{-}$. Observons que la fonction donnée $1 / \tau \bar{\tau}^{\prime}$ est continue sur $L$ et vérifie la condition do Hölder avec l'exposant $h_{L}$ et que la fonction donnée $c(\tau)$ est discontinue sur $L$, de classe $\mathfrak{S}_{\alpha}^{h^{\prime}}\left(c_{k_{1}}, \ldots, c_{k_{q}}\right)$, où $h^{\prime}=\min \left(h, h_{L}\right)$.

On voit que l'indice du problème homogène est nul, done, d'après la théorie du problème non homogène discontinu de Riemann (voir travail [1]), la solution générale du problème (20) aura la forme

$$
\Omega_{0}(z)=\omega(z)+A \omega_{1}(z)
$$

où $\omega(z)$, de classe $H_{a}^{h^{\prime}}\left(c_{k_{1}}, \ldots, c_{k_{q}}\right)$, est une solution particulière du problème $(20), \omega_{1}(z)$ une solution particulière du problème homogène continu de Riemann

$$
\mathrm{re}\left[\frac{\tau^{\prime}}{\tau} \omega_{1}(\tau)\right]=0
$$

et $A$ est une constante réelle arbitraire. On la choisit de façon que la fonction re $\left[\Omega_{0}(z)\right]$ soit nulle à l'infini et on arrive, d'après la formule (19), à la fonction unique $\mu(\tau)$ de classe $\mathfrak{S}_{\alpha}^{h^{\prime}}\left(c_{k_{1}}, \ldots, c_{k_{q}}\right)$ et à la constante réelle unique $C$, correspondant à la fonction donnée $\Phi(z)$. En substituant la fonction $\mu(\tau)$ et la constante $C$ obtenues dans l'intégrale (18), on obtient la fonction $\Phi(z)$ donnée. L'existence de la représentation (17) est donc démontrée.

Théorìme 9. Si la dérivée d'ordre $m(\geqslant 1)$ d'une fonction $\Phi(z)$, holomorphe dans le domaine $S^{+}$, appartient $\grave{a}$ la classe $H_{a}^{h}\left(c_{k_{1}}, \ldots, c_{k_{q}}\right)$ :

$$
\Phi^{(m)}(z) \epsilon H_{\alpha}^{h}\left(c_{k_{1}}, \ldots, c_{k_{q}}\right)
$$

relativement aux points de discontinuité $c_{1}, \ldots, c_{p}$, on peut représenter d'une façon unique cette fonction par la formule intégrale

$$
\Phi(z)=\int_{L} \mu(\tau)\left(1-\frac{z}{\tau}\right)^{m-1} \log \left(1-\frac{z}{\tau}\right) d \sigma+\int_{L} \mu(\tau) d \sigma+i C,
$$

oì $\mu(\tau)$ est une fonction réelle de classe $\mathfrak{S}_{a}^{h^{\prime}}\left(c_{k_{1}}, \ldots, c_{k_{q}}\right)$ et $h^{\prime}=\min \left(h, h_{L}\right)$. $C$ est une constante réelle et l'on a choisi la branche continue de la fonction logarithmique qui s'annule au point $z=0$, contenu à l'intérieur de $\mathrm{S}^{+}$.

Tout d'abord remarquons que, en vertu du théorème 3 , les dérivées d'ordre inférieur à $m$ et la fonction $\Phi(z)$ elle-même ont des valeurs limites déterminées en tout point $t$ de la ligne $L$, même aux points de discontinuité $c_{y}$. En outre, les fonctions limites vérifient une simple condition de Hölder.

En s'appuyant sur les propriétés, précédemment citées, des fonctions des classes $\mathfrak{S}_{a}^{h}$ et $H_{\alpha}^{h}$, on peut démontrer l'existence de la fonction $\mu(\tau)$ et de la constante $C$, correspondant d'une façon unique à la fonction donnée $\Phi(z)$, en suivant un raisonnement tout à fait analogue à celui qui à été donnée d'une façon très précise et complète par Vécoua (voir [2] et [3]).

Sans reproduire cette démonstration, signalons seuloment que, l'existence d'une fonction $\mu(\tau)$ de classe $\mathfrak{S}_{\alpha}^{h^{\prime}}\left(c_{k_{1}}, \ldots, c_{k_{q}}\right)$ étant admise, nous tirons de la formule (23) l'expression suivante pour la dérivée d'ordre $m$

$$
\begin{aligned}
\Phi^{(m)}(\boldsymbol{z}) & =(-1)^{m}(m-1) ! \int_{\mathcal{L}} \frac{\mu(\tau) d \sigma}{\tau^{m-1}(\tau-z)} \\
& =(-1)^{m}(m-1) ! \int_{L} \frac{\mu(\tau) \bar{\tau}^{\prime} d \tau}{\tau^{m-1}} \frac{(\tau-z)}{(\tau-z)}
\end{aligned}
$$


En faisant tendre le point $z$ vers un point arbitraire $t \neq c_{y}$ sur $L$, on aura, d'après les formules de Plemelj, pour la fonction $\mu(\tau)$ une équation intégrale de la forme

$$
\mu(t)+\frac{1}{\pi i} \int_{L} \frac{t^{m-1} t^{\prime} \mu(\tau) d \sigma}{\tau^{m-1}(\tau-t)}=\frac{t^{m-1} t^{\prime} \Phi^{(m)}(t)}{(-1)^{m}(m-1) ! \pi i} .
$$

D'après la théorie de Vécoua, cette équation est équivalente à l'équation intégrale réelle

$$
\mu(t)+\int_{\dot{L}} \mathrm{re}\left[\frac{t^{m-1} t^{\prime}}{\pi i \tau^{m-1}(\tau-t)}\right] \mu(\tau) d \sigma=\mathrm{re}\left[\frac{t^{m-1} t^{\prime} \Phi^{(m)}(t)}{(-1)^{m} \pi i(m-1) !}\right]
$$

dont le noyau admet une faible singularité. Elle met en évidence le fait que la fonction $\mu(\tau)$ appartient à la classe $\mathfrak{S}_{\alpha}^{h^{\prime}}\left(c_{k_{1}}, \ldots, c_{k_{q}}\right)$ si la dérivée $\Phi^{(m)}(z)$ de la fonction donnée appartient à lä classe $H_{a}^{h}\left(c_{k_{1}}, \ldots, c_{k_{q}}\right)$.

\title{
Travaux cités
}

[1] W. Pogorzelski, Problèmes aux limites discontinues dans la théorie des fonotions analytiques (résumé), Bulletin de l'Académio Polonaise des Sciences, Sér. des sci. math., astr. et phys. 7 (1959), p. 311-317, ou: Journal of Mathematics and Mechanics, Indiana University, 1960.

[2] I. N. Vécoua, Sur une nouvelle représentation intégrale des fonotions analy. tiques (en russe), Comptes Rendus de l'Académie Géorgienne des Sciences, t. II, No 6 (1941), p. $477-484$

[3] N. J. Muskhelishvili, Singular integral equations, P. Noordhoff, N. V. Groningen - Holland 1953.

INSTYTUT MATEMATYCZNY POLSKIEJ AKADEMII NAUK

INSTITUT MATHEMATIQUE DE L'ACADEMIE POLONATSE DES SCIXNCES

Reçu par la Rédaction le 14. 10. 1959

\section{POLONICI MATHEMATICI}

IX (1960)

\section{Le trièdre de Frenet aux points d'inflexion d'une courbe}

\author{
par S. GoÆąB (Kraków)
}

Les démonstrations de l'existence des notions classiques de la géométrie différentielle des courbes et des surfaces exigent certaines hypothèses. Ces hypothèses présentent, d'une part, le caractère d'une certaine régularité de la représentation analytique de l'être envisagé, d'autre part, elles possèdent le caractère de certaines inégalités servant à exclure les points singuliors qui peuvent se présenter malgré la haute régularité de la représentation analytique. A titre d'un simple exemple, rappelons que pour assurer l'existence d'une tangente au sens ordinaire en un point donné (le contingent au sens de M. G. Bouligand doit contenir en ce point deux rayons opposés) il suffit de supposer que la représentation analytique (sous forme vectorielle) de la courbe

$$
\boldsymbol{r}=\boldsymbol{r}(u)
$$

(où $\boldsymbol{r}$ désigne le rayon-vecteur du point mobile de la courbe et $u$ est un paramètre arbitraire) est de classe $C_{1}^{*}$, e'est-à-dire que la dérivée $d \boldsymbol{r} / d u$ est continue et qu'on a, en outre,

$$
|d \boldsymbol{r} / d u|>0 .
$$

L'hypothèse $C_{1}$, admise sans que la condition supplémentaire (2) soit satisfaite, n'exclut pas l'existence d'un point singulier où la tangente peut ne pas exister. D'une façon analogue, pour l'existence du trièdre de Frenet (dans ce but il suffit qu'en plus de la tangente existe la normale principale) il suffit que la représentation soit de classe $C_{2}^{* *}$, c'est-à-dire que la seconde dérivée $d^{2} \boldsymbol{r} / d u^{2}$ soit continue et que les vecteurs $d \boldsymbol{r} / d u$, $d^{2} \boldsymbol{r} / d u^{2}$ soient linéairement indépendants.

Si la courbe (1) est de classe $C_{1}^{*}$ et $C_{2}$, mais n'est pas de classe $C_{2}^{* *}$ au point $u=u_{0}$, e'est-à-dire si le produit vectoriel

$$
\frac{d \boldsymbol{r}}{d u} \times \frac{d^{2} \boldsymbol{r}}{d u^{2}}
$$

